\title{
Gradual Return to Play: Potential Role of Neurotoxicity Biomarkers in Assessment of Concussions Severity
}

Svetlana A Dambinova*, Richard L Sowell and Joseph C Maroon

Kennesaw State University, Kennesaw, Georgia, USA

University of Pittsburgh Medical Center, USA

\begin{abstract}
This mini-review analyzes advantages and limitations of current methods of assessment of cerebral concussions considering functional, structural and metabolic factors in decision making for return to play. Novel neurotoxicity biomarkers, AMPA and NMDA receptor peptide/antibodies are proposed for evaluation of subtle brain injury following concussions. Neurotoxicity biomarkers can now be detected in the blood and are associated with severity of concussion and transient or persistent changes in the brain including diffuse axonal injury and microvascular dysfunction (edema formation).

We surveyed 84 students $(20.5 \pm 2.5$ years $)$ participating in contact-sports and enrolled at Kennesaw State University. Based on neurotoxicity biomarkers values and diminished ImPACT scores, 18 athletes with concussions were selected for longitudinal assessment (1.5 year). Within the study, values of four neurotoxicity biomarkers decreased to normal in $11(61 \%)$ concussed athletes while seven subjects maintained at least one acute and chronic biomarkers elevated reflecting structural changes in the brain defined by 3T DTI.

It was demonstrated that neurotoxicity biomarkers in conjunction with neurocognitive testing might improve diagnostic certainty of suspected concussions. Additionally the use of biomarkers may provide valuable information on severity of concussions and help select subjects (about $2 \%$ ) for advanced neuroimaging. Athletes with abnormal levels of neurotoxicity biomarkers and structural changes on DTI should be withheld from contact sports and be considered for therapeutic intervention and treatment to protect the brain from further neurological complications.
\end{abstract}

Keywords: Concussions; Diagnosis; Brain biomarkers; Neurocognitive testing; Neuroimaging

\section{Introduction}

The CDC estimates from 1.6 to 3.8 million concussions occur each year in the United States [1]. Within 2001-2005, about 1.0 million emergency department (ED) visits with nonfatal brain injuries were registered leaving up to $74 \%$ with sport-related concussions unobserved and under reported. The first problem in assessment of concussions is being aware of the culture of denial in athletes and also in the military [2]. The second challenge concerns the difficulties in diagnosis of sport-related concussions and mild traumatic brain injury (TBI) with aspectrum of subclinical signs that may evolve over hours or days after the impact [3]. Additionally, neuroimaging is usually normal and not helpful except to rule out an intracranial hemorrhage or contusion. There is a practical need for rapid, reliable, and affordable biomarkers that can improve diagnostic accuracy of concussions, define severity and aid in gradual return to play, work or duty.

There are three independent types of biomarkers: functional, structural and metabolic. Functional biomarkers are associated with cognitive skills and are closely linked to the function of particular cerebral areas, neural pathways, or cortical networks. Neurocognitive testing assesses anatomical and physiological brain functions related to orientation, memory, reaction time, processing of information and cognitive efficiency. Structural biomarkers assess the topography of brain architecture, its pathways, and connectivity. Fiber tracts and micro vascular disruption may be visualized by advanced magnetic resonance imaging (MRI) modalities (susceptibility weighted imaging, MR spectroscopy, diffusion tensor imaging, and functional MRI). Metabolic brain biomarkers derive from genomic, proteomic and degradomic cascades. The key molecular biomarkers currently include - but not limited to - proteins, enzymes, protein degradation products and, more recently microRNAs that reflect glial and neuronal dysfunction and immune system activation after brain injury [3-5].
Molecular biomarkers used in conjunction with neurocognitive testing and advanced neuroimaging may provide a dynamic and powerful approach to understanding the spectrum of transient and persistent changes in nervous tissue after concussions. This mini-review analyzes advantages and limitations of current methods of assessment of concussions including functional, structural and metabolic methods for decision making return to play criteria.

\section{Functional Biomarkers of Concussions}

The Consensusin Concussion Sport Group endorsed neurocognitive testing as the "cornerstone" of concussion management [4]. Current neurocognitive tests for assessment of concussions are offered in different settings: at a training facility, in emergency departments, on site (at stadium), and in military settings- on the battle field. These functional biomarkers are widely used in making decision for return to play despite different approaches to mild TBI severity scoring and disagreements in procedure standardization [6-8]. There are several practical approaches for rapid and convenient neuropsychological assessment of concussions proposed.

Immediate Post-Concussion Assessment and Cognitive Testing (ImPACT) is the most-widely used computerized neurocognitive evaluation system for comprehensive management of concussions

*Corresponding author: Svetlana A Dambinova, Kennesaw State University, Kennesaw, Georgia, USA, E-mail: sdambino@kennesaw.edu

Received April 01, 2013; Accepted April 29, 2013; Published May 01, 2013

Citation: Dambinova SA, Sowell RL, Maroon JC (2013) Gradual Return to Play: Potential Role of Neurotoxicity Biomarkers in Assessment of Concussions Severity. J Mol Biomark Diagn S3: 003. doi:10.4172/2155-9929.S3-003

Copyright: (C) 2013 Dambinova SA, et al. This is an open-access article distributed under the terms of the Creative Commons Attribution License, which permits unrestricted use, distribution, and reproduction in any medium, provided the original author and source are credited 
to guide decision making for return to play and to safely manage concussions in non-athletes [9-11]. ImPACT records demographic information utilizes six neuropsychological tests and assesses postconcussion symptoms. A composite score is then generated that assesses verbal memory, visual memory, reaction time, visual motor processing speed and impulse control. ImPACT could be administered in the ED setting with results available in under 25 minutes; however most testing occur 3-10 days post-injury (http://impacttest.com/about/ background).

Display Enhanced Testing for Concussion and mTBI system (DETECT) has been developed in response to the lack of an efficient neuropsychological testing in the "field" [12]. The device uses a headset designed to filter out noise and visual distractions. Then a seven-minute battery of cognitive tests is administered similar to the computer-based neurocognitive ImPACT. It might help trainers and clinicians better detect concussions without removing players from the stadium. However this device is still under the evaluation for validity and reliability.

\section{Eye-tracking test}

Eye movements are frequently impaired after concussion. If the recovery path of eye movement function differs from the normal after mild TBI it may be indicative of ongoing cerebral impairment [13]. Eye-tracking technology is an objective concussion test that can be used in combat settings (critical diagnosis in under a minute). The prototype contains a high-resolution camera (video-oculography) that records as the subject visually tracks a target that moved through a circular trajectory [14]. It was demonstrated that gaze error variability significantly correlated with attention and working memory measures in neurocognitive testing.

Head Impact Telemetry (HIT) system technology allows for easy and accurate real-time monitoring of on-field head impacts. The MX Encoder, part of the patented technology, provides on-board electronics in the helmet that monitors and records impacts while in use. The system uses accelerometers to measure the location, magnitude, duration, and direction of head impacts, impact accumulations, and then transmits that information wirelessly to the sideline (http://www. riddell.com/innovation/hits-technology).

\section{Structural Biomarkers of Concussions}

The human brain is the most complex living structure and is divided into cortical and subcortical areas responsible for various executive functions. The brain of an adult human with over 100 billion neurons, $165,000 \mathrm{~km}$ of myelinated nerve fibers and some 0.15 quadrillion synapses presents a combination of pathways forming a three-dimensional cerebral architecture [15]. Advanced MRI methods have demonstrated the ability to detect and localize with high resolution pathologic and pathophysiologic consequences of mTBI.

Diffusion tensor imaging (DTI) is a relatively new MRI modality that capitalizes on the diffusion of water molecules for brain imaging and detects axonal injury and abnormalities in white matter connectivity after mild TBI [16]. Compared with traditional neuropsychological testing and conventional imaging techniques, DTI imaging was more accurate in differentiating mild TBI from controls at 24 hours and 1 month post injury [17-19]. DTI based tractography is another promising method for probing alterations in the trajectories of the fiber tracts (bundles) in white matter after mild TBI [16]. Results of eye-tracking tests significantly correlated with the mean fractional anisotropy (FA) the DTI parameter that measures the microstructural integrity of white matter tracts [14].
Functional magnetic resonance imaging (fMRI) measures changes in blood oxygen level locally in response to neuronal activity. One study examined high school football players and revealed a group of players who exhibited no clinically-observed symptoms associated with concussions [20]. However these subjects had significantly higher numbers of head collision detected by HIT system, demonstrated impairments in primarily visual working memory assessed by ImPACT, and showed altered activation in dorsolateral prefrontal cortex defined by fMRI [20]. It was also suggested that fMRI integrated with DTI may be more suitable for correlating neuropsychological differences, including cognitive processing and outcomes from mild TBI [21,22].

Proton (1H) magnetic resonance (MR) spectroscopy has the advantage of measuring brain metabolism in vivo, and is able to detect various biochemical processes of brain injury such as the loss (or dysfunction) of neuronal cells. TBI is known to induce changes in $\mathrm{N}$-acetyl aspartate (NAA), the second most concentrated molecule in the brain after the amino acid glutamate that is located and synthesized in the mitochondria of neurons [23]. Decreased NAA concentrations were detected in the whole brain [24], in the corpus callosum of student athletes [25], in the prefrontal and motor cortex of football players [26] and in the semi-acute and chronic phase of mild TBI. Global white matter NAA concentrations showed sensitivity to the mild TBI sequelae associated with common post-concussive symptoms in individuals with mostly normal neuroimaging as well as normal Glasgow Coma Scale (GCS) scores [27].

Susceptibility weighted imaging (SWI) is a high resolution venography method that is sensitive to deoxyhemoglobin in the acute stage, extracellular methemoglobin in the subacute stage, and hemosiderin in the chronic stage allowing the detection of cerebral hemorrhages [28]. The hemosiderin was detected on a 3T MRI scanner in person with uncomplicated mild TBI using SWI modality that would likely be missed by $1.5 \mathrm{~T}$ or $3.0 \mathrm{~T}$ MRI conventional sequences [29]. Another study included SWI, DTI and MR spectroscopy modalities showed that collectively three advanced MRI could detect abnormalities in $80 \%$ patients with mild TBI [30]. SWI detected a parenchymal injury, DTI showed diffused axonal injury in major white matter tracts (the splenium of corpus callosum and superior corona radiata), and MR spectroscopy measured abnormally high glutamine (Gln) signals in fronto-parietal subcortical white matter.

\section{Current Molecular Biomarkers for Mild TBI}

Pathological changes on molecular level that occur in the nervous tissue soon after injury can be detected in the blood by biochemical tracers or biomarkers [2]. The use of a brain biomarker or panel of biomarkers that may rapidly assess suspected concussion would be invaluable in a contact sport setting. It would improve diagnostic certainty of brain injury and lead to immediate and prompt treatment.

Metabolic, immunologic and excitotoxic phenomena all combine to cause damage in the brain after a cerebral concussion. Blaylock and Maroon [31] recently described an immune-excitotoxic reaction as a unifying neuroinflammatory hypothesis for post concussion syndrome, post-traumatic stress disorder (PTSD) and chronic traumatic encephalopathy (CTE). This reaction leads to the disruption of axons and synapses and depending on the location and acuteness of an injury a number of biomarkers might be detected in biological fluids (Figure 1).

The molecular mechanisms of brain injury involve several multisystem processes: neurotoxic degradation of neuroreceptors, micro 
Citation: Dambinova SA, Sowell RL, Maroon JC (2013) Gradual Return to Play: Potential Role of Neurotoxicity Biomarkers in Assessment of Concussions Severity. J Mol Biomark Diagn S3: 003. doi:10.4172/2155-9929.S3-003

Page 3 of 11

vascular endothelium dysfunction, and immune response activation that result in primary [31] and secondary events. There is a number of central nervous system (CNS)-born and systemic blood-born enzyme families, including serine and cysteine proteases, matrix metalloproteinases which initiate a proteomic or degradomic cascades due to impact. Key metabolites of proteolytic cascades can be classified according to pathological conditions; i.e. ischemic, hemorrhagic, or axonal injury associated with abnormal spiking activity. Recent reviews of the literature showed a number of molecular biomarkers derived from neurons and glial cells which are proposed for assessment of mild TBI $[3,4]$.

\section{Glial markers}

$S 100 B$ protein is predominantly expressed in astrocytes that can be detected in human CSF and serum and is considered as an indicator of astrocytic damage after moderate-to-severe brain injury that reflects blood-brain barrier (BBB) disruption [3]. S100B is proposed to be a biomarker for ruling out the injury. However $\mathrm{BBB}$ appears mostly intact after suspected concussions/mild TBI. Recent clinical studies demonstrated that elevated levels of auto-antibodies against S100B were detected only after repeated sub-concussive events or more severe TBI characterized by BBB disruption [32]. Serum levels of S100B autoantibodies also predicted persistence of MRI-DTI abnormalities which in turn correlated with cognitive changes.

Glial Fibrillary Acidic Protein (GFAP) is a monomeric intermediate filament protein expressed by astrocytes [33] and is considered as a biomarker of hematoma/hemorrhage released after intracerebral or subarachnoid hemorrhage and TBI [3,34]. Elevated concentrations of GFAP were detected in Olympic boxers after 47 bouts compared to controls [35]. Studies in adults [36] have demonstrated that breakdown product of GFAP (GFAP-BDP) values allowed differentiating mild TBI subjects with GCS scores of 15 from uninjured controls (area under ROC curve of 0.88). However GFAP concentrations were not discernible in $63 \%$ patients with mild TBI and were abnormally high only in patients with an abnormal CT [37].

\section{Neuronal markers}

Neuron-specific enolase (NSE) a glycolytic protein is primarily located in the neuronal cytoplasm. Significant elevations of NSE concentration have been observed following TBI that correlated with severity of the injury $[3,38]$. Based on the available research evidence, it was concluded that NSE does not have adequate sensitivity (40\%-89\%) to assess acute mild TBI due to relatively small increases in NSE levels after concussions $[3,4]$.

Tau protein is a microtubule-associated structural protein located within axons. Studies focused on detection of Tau protein showed no difference between patients in the acute phase of mild TBI and control subjects [39], demonstrated poor predictive power of outcome [40], and does not predict post-concussion syndrome [39]. However recent studies of biomarkers in CSF and plasma depicted significantly higher

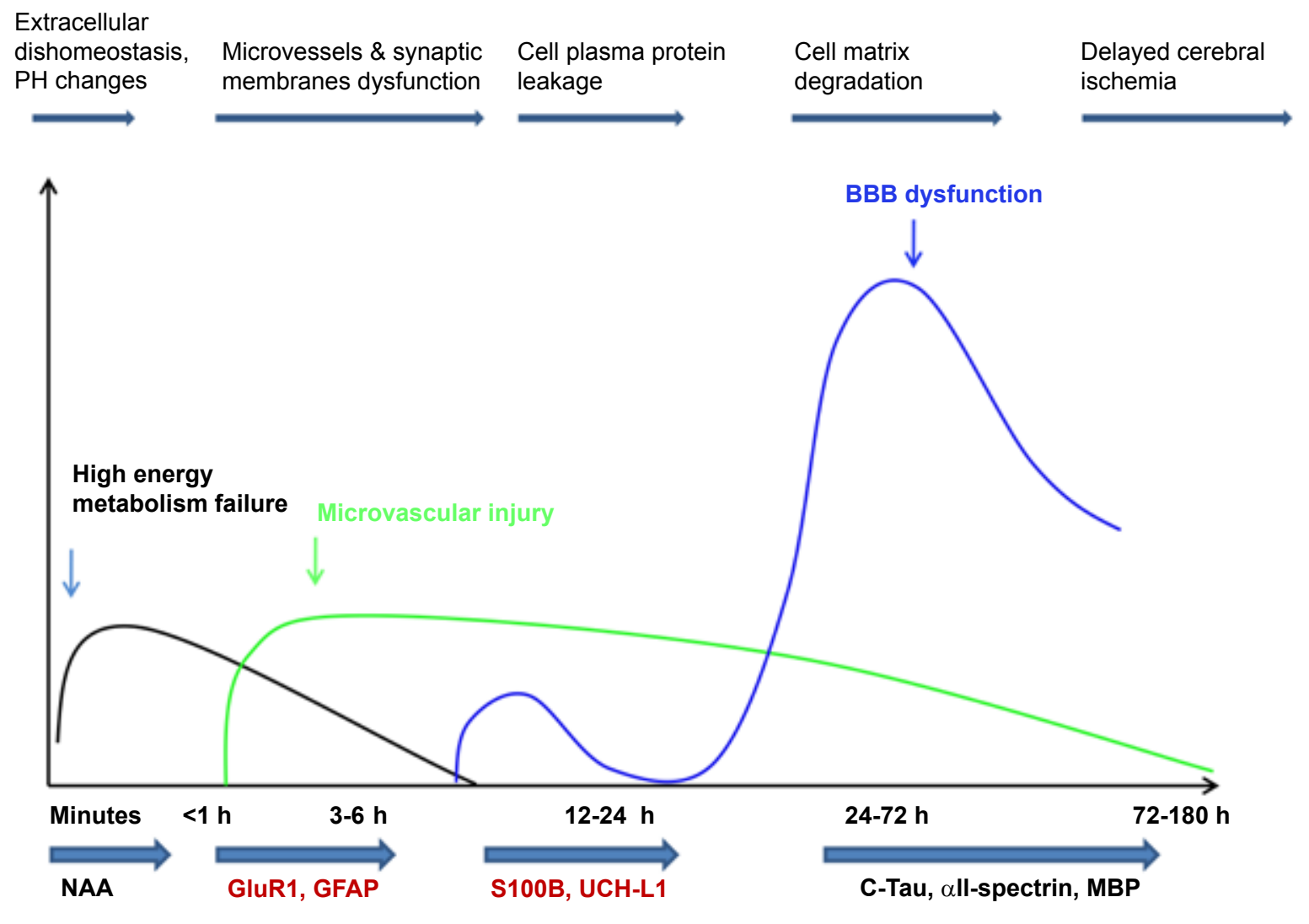

Timeline of biomarkers release into bloodstream

Figure 1: Mechanisms involved in development of subtle brain injury after mild TBI and timely release of corresponding biomarkers 
Tau protein concentrations in Olympic boxers $(n=30)$ after 47 bouts compared to controls $[35,41]$. The abnormal deposits of Tau protein were found in glia and neurons of postmortem slices of professional athletes with chronic traumatic encephalopathy developed after multiple concussions [42].

The neuron-specific cytoplasmic ubiquitin C-terminal hydrolase $(U C H-L 1)$ is a proteolytically stable and abundant protein which is found exclusively in neurons. Studies in adults [43] have demonstrated that UCHL1 values distinguished moderate and severe TBI from uninjured controls with sensitivity of $95 \%$ within $4 \mathrm{~h}$ after the injury [43]. The same marker assessed acute mild TBI persons with GCS scores of 15 with area under ROC curve of 0.87 . However in children $(<15$ years of age) in acute phase ofmild TBI $(n=11)$, UCHL1 showed the lack of the differentiation between injury and controls [38].

$\alpha I I-S p e c t r i n$ is primarily found in neurons and is abundant in axons and presynaptic terminals. It is processed into break down products (SBDP) of 145,150 , and $120 \mathrm{kDa}$. SBDP120 is produced by proteolytic cleavage with caspase- 3 , and is primarily associated with apoptosis. SBDP145 and SBDP150 are produced by cleavage with calpain and are primarily associated with necrosis. There were no detectable changes in serum concentrations of SBDP145 found in pediatric patients in acute phase of mild TBI [38]

\section{Novel Neuronal and Microvessel Biomarkers}

Glutamate receptors (GluRs) mediate a majority of fast excitatory synaptic neurotransmission in the mammalian brain and their functions are absolutely critical for human executive functions including thinking, consciousness, memory, and learning. Most importantly, these neuroreceptors regulate long- and short-term synaptic plasticity through adaptation via alterations in gene expression [44]. The latter is a major component of human behavioral adaptation to different environmental conditions.

GluRs are a family of approximately 25 proteins comprising channel activated ionotropic and G-protein regulated metabotropic receptors, both of which initiate secondary messengers and some of them are located on microvascular surfaces [45]. Ionotropic GluRs also mediate neuron-glial contacts and glutamatergic neurotransmission [46]. More than $80 \%$ of cortical, subcortical, and spinal cord neuronal communications are dependent on structure, functions, and location of GluRs [47]. There are three major ionotropic GluRs subtypes: a-amino-3-hydroxy-5-methyl-4-isoxazolepropionic acid (AMPA) [48], N-methyl-D-aspartate (NMDA) [49] and kainite receptors [50], which are categorized by structural similarity, electrophysiological functioning, and selective pharmacological properties. NMDA receptors mainly control $\mathrm{Ca}^{2+}$ channels, while non-NMDA (AMPA and kainite) receptors regulate permeability of all three ion channels $\left(\mathrm{K}^{+}\right.$, $\mathrm{Na}^{+}$, and $\mathrm{Ca}^{2+}$. Molecular diversity of GluRs genes encoding various receptor subunits is responsible for pharmacological and functional heterogeneity. A number of reviews are available on the role of GluRs and their implication on the pathology of neuronal injury [51].

It is necessary to note that the $\mathrm{N}$-terminal sequences of these ionotropic receptors can determine protein structure, function, localization, and renewal rates.Any subtle changes to protein sequences by selective proteases generating a new $\mathrm{N}$-terminal peptide or protein, sometimes with only a few amino acids truncated, may have a dramatic effect on protein function and can serve as initiating or key steps in the proteolytic control of signaling cascades [52]. That is important issue to consider in search of location-specific biomarkers and pharmaceutical drug - diagnostics co-development.
AMPA receptors are primarily located on axons and oligodendrocytes [53,54] and distributed in the forebrain of subcortical pathways including the hippocampus, amygdala,thalamus, hypothalamus, and brain stem [55-57]. This architecture supports the noncortical nature of subtle brain injury. These regions of the brain are predictable sources of biomarkers given the functional spatialtemporal coherence, developmental pathways, and cerebral plasticity subjected to coup-countercoup mild brain injury [58].

$\mathrm{N}$-methyl-D-aspartate(NMDA) receptors predominate in forebrain structures with considerable representation in the cortex [59]. The NMDA receptor plays an essential role in cerebral plasticity, the ability of the brain to change in response to external stimuli such as learning and memory. It was shown that (NMDA) excitatory receptors which are located on the surface of microvessels, regulate vasoconstriction/ vasodilatation, and subtle neurovascular dysfunctions [45,60]. An important aspect of NMDA receptor function (NR2 subunits) is that it can regulate cell damage or survival under pathological conditions such as edema/cerebral ischemia and TBI [34].

There were reported AMPA receptor mechanosensitivity and phosphorylation to traumatic stretch on a molecular level $[61,62]$ Alterations of AMPA and NR2 subunit of NMDA receptor expressions, densities in the brain, and subsequent trafficking of degradation products in the blood stream through the compromised BBB after mild cerebral injury in animals and humans were investigated [63-65].

NMDAR and AMPAR are key components that control the neurotoxicity cascade by engaging in immediate and cumulative sequelae following TBI with following acute and chronic changes [34]. On the basis of molecular, biochemical, and immunohistochemical investigations, the N-terminal domain of AMPA receptors was proposed as a biomarker of initial necrotic events. The NR2 peptide, a N-terminal fragment of NMDA receptors is a biomarker of delayed ischemic events following acute and sub-acute mild TBI [66,67]. The excitatory neurotransmitter receptors, especially those of extracellular $\mathrm{N}$-terminal location may be promising biomarkers for assessment of subtle brain injury in acute and chronic phases.

\section{Acute and sub-acute concussions and transient changes in brain}

Primary injury to the brain causes mechanical damage and energy failure in parenchymal cells and endothelia that comprise BBB. Traumatic injury causes brain peptides to be released continuously into the bloodstream through the compromised BBB within hours to days (acute phase) after the impact, while most proteins enter biological fluids relatively late, up to several weeks after the injury (subacute phase) [68]. In general, initial BBB leakage is linked to trafficking of small or middle-size peptides that may have different life times in blood and CSF [69]. Some peptides can be rapidly digested by enzymes, whereas others with active epitopes can activate immune response and produce antibodies that might serve as an evidence of asymptomatic or subclinical events [70]. The monitoring of these antigens in biological fluids allows one to define an early pathological cascade after acute and sub-acute brain injury.

Recently at Kennesaw State University feasibility study for the diagnostic potential of the AMPAR peptide assay in conjunction with neuropsychological testing and neuroimaging to differentiate concussions from healthy controls were examined [6]. The significant sensitivity/specificity of AMPAR peptide ( $91 \%$ and $92 \%$ respectively) was determined to assess acute and semi-acute concussions in college 
Citation: Dambinova SA, Sowell RL, Maroon JC (2013) Gradual Return to Play: Potential Role of Neurotoxicity Biomarkers in Assessment of Concussions Severity. J Mol Biomark Diagn S3: 003. doi:10.4172/2155-9929.S3-003

Page 5 of 11

athletes [64,65]. Also in athletes with multiple concussions, worse ImPACT scores correlated with abnormal levels of the biomarker and increased AMPAR peptide values $(2.0-12.0 \mathrm{ng} / \mathrm{mL})$ were associated with minor findings on magnetic resonance imaging [65].

\section{Subjects and procedures}

We surveyed 84 contact-sport athletes $(20.5 \pm 2.5$ years $)$ enrolled at Kennesaw State University between September 2011 to March 2012 [65]. Based on neurotoxicity biomarkers values and diminished ImPACT scores, 18 out of 33 athletes with concussions presented within 1-2 weeks after the impact were selected for longitudinal assessment (1.5 year). These subjects were followed up with neurocognitive testing (ImPACT, version 2.1) and neurotoxicity biomarkers assays. The dynamic changes of neurotoxicity biomarkers were analyzed in three concussed subjects that were assigned for neurological examination and MRI [65]. One athlete out of three subjects underwent 3T DTI imaging (Siemens MAGNETOM Spectra 3T Skyra) at DeKalb Medical, Decatur, GA.

\section{Samples Collection and Assays}

At the time of enrollment, each study participant had a single blood draw by venipuncture into vacuum tubes (Becton Dickinson) containing either sodium ethylene diamine tetraacetic acid (plasma) or gel separator (serum). Selected subjects had three additional blood samples drawn during follow up-visits (within 1,3 and 15 months of the first sample withdrawal). Plasma samples for AMPAR and NR2 peptide assays using magnetic particles (MP)-ELISA method [65,71] and serum samples for AMPAR and NR2 antibody assays by ELISA technique $[64,66,70]$ were assessed.

\section{Results}

The basic evaluation of contact-sport athletes included ImPACT test and neurotoxicity biomarkers assays performed for KSU Sports Club athletes after the fall season of 2011 [65]. Only two athletes within selected group had records of concussion in the past 6 months. In the next 15 months of study values of AMPAR peptide biomarker decreased close to normal $(0.07-0.9 \mathrm{ng} / \mathrm{mL})$ in $11(61 \%) \%$ out of 18 concussed athletes that showed passable ImPACT scores (Figure 2A) and returned to play. In longitudinal assessment (up to 1.5 year), seven subjects with concussions maintained AMPAR peptide values increased above cut off of $0.4 \mathrm{ng} / \mathrm{mL}$. Abnormal values of the biomarker in five
A

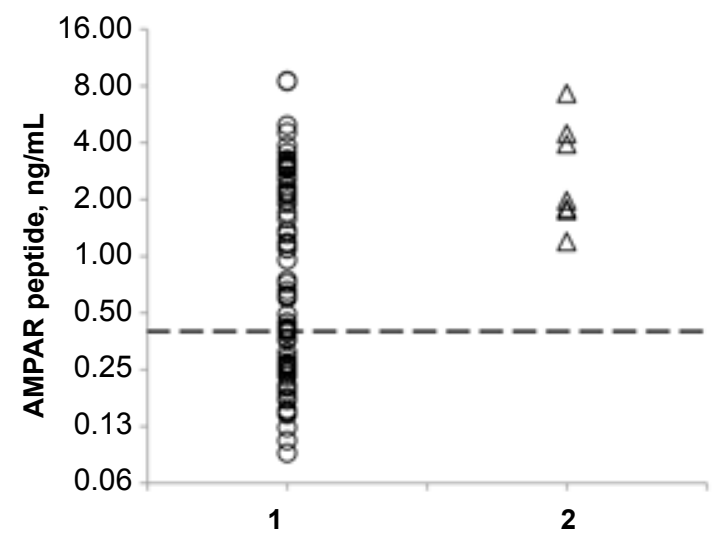

C

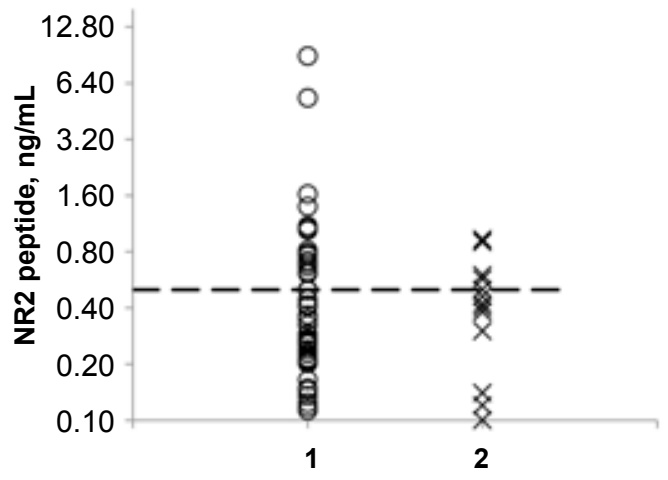

B

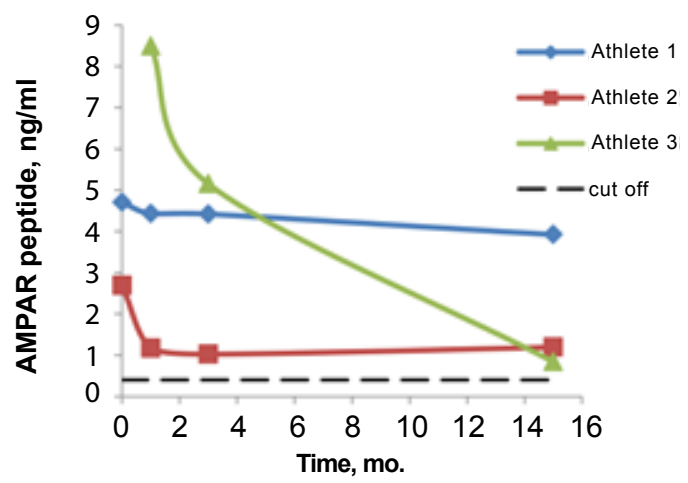

D

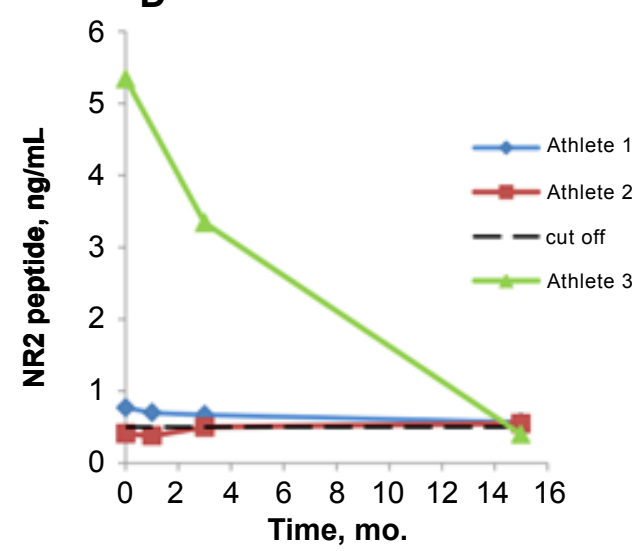

Figure 2: Distribution of AMPAR plasma concentrations in A: (1) all enrolled athletes $(n=84)$ and (2) not recovered from prior concussion athletes ( $n=7$ ). Dynamic changes of plasma AMPAR peptide for three subjects (B); Distribution of NR2 plasma concentrations in $C$ : (1) all enrolled athletes ( $n=84)$, (2) subjects returned for longitudal study $(n=18)$. Dynamic changes of plasma NR2 peptide for three subjects (D). Dotted lines show corresponding cut offs: $0.4 \mathrm{ng} / \mathrm{mL}$ for AMPAR peptide and $0.5 \mathrm{ng} / \mathrm{mL}$ for NR2 peptide. 
Citation: Dambinova SA, Sowell RL, Maroon JC (2013) Gradual Return to Play: Potential Role of Neurotoxicity Biomarkers in Assessment of Concussions Severity. J Mol Biomark Diagn S3: 003. doi:10.4172/2155-9929.S3-003

Page 6 of 11

subjects accompanied with decreased visual memory composite score (5-50\%) assessed by ImPACT test performed in 2013.

Based on poor ImPACT scores and abnormally high AMPAR peptide values, 3 athletes with multiple concussions were recommended for neurological consultation and MRI. There were no abnormal signals in the typical locations for axonal shearing damage in $1.5 \mathrm{~T}$ images for all three subjects [70]. No significant motor, sensory, or cognitive deficits on their neurological exams were noted. These athletes had a history of multiple concussions with persistent headache and mild cognitive dysfunction. The symptoms appeared to be resolved within 3 weeks for two subjects; however AMPAR peptide values maintained elevated up to 15 months with different profiles (Figure 2B). In one subject (athlete \#1) the biomarker did not reach control value during the entire study.

\section{Case study}

Athlete \#1, a 22-year-old male student presented with a history of semi-acute concussion on 11/5/2011. He was playing ice hockey and struck the back of his head while wearing a helmet. There was no loss of consciousness but he was lying on the ice, unable to move. He was temporarily removed from the game. He subsequently passed a sideline evaluation and returned to the game. The next day he began having headaches, impaired balance, nausea, and sensitivity to light and noise. He stayed out of school for 4 weeks with persistent headaches and sleeplessness. 3T DTI scans for this subject showed significant white matter changes with decreased fractional anisotropy (FA) compared to non-injured subject scan (Figure 3). There were a number of white matter fiber tracts absent in frontal and parietal areas of brain indicating the severity of concussion and need in treatment during the recovery.

Considering DTI data and AMPAR peptide monitoring results for athlete \#1, we may suggest that athletes \#2 and \#3 that AMPAR peptide values maintained abnormally high within 3 months could have transient changes in the brain that may be detected by 3T fMRI if performed within 3-6 months after concussion.
A

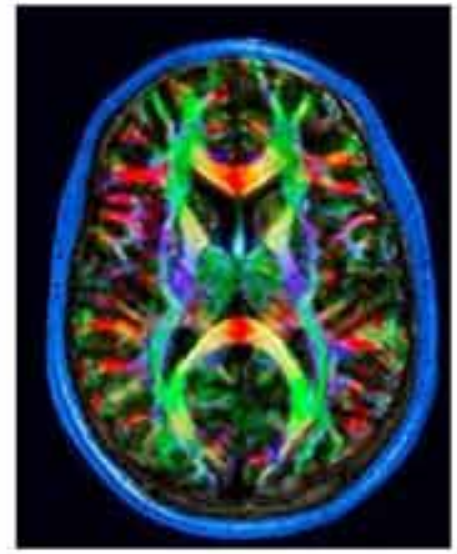

C

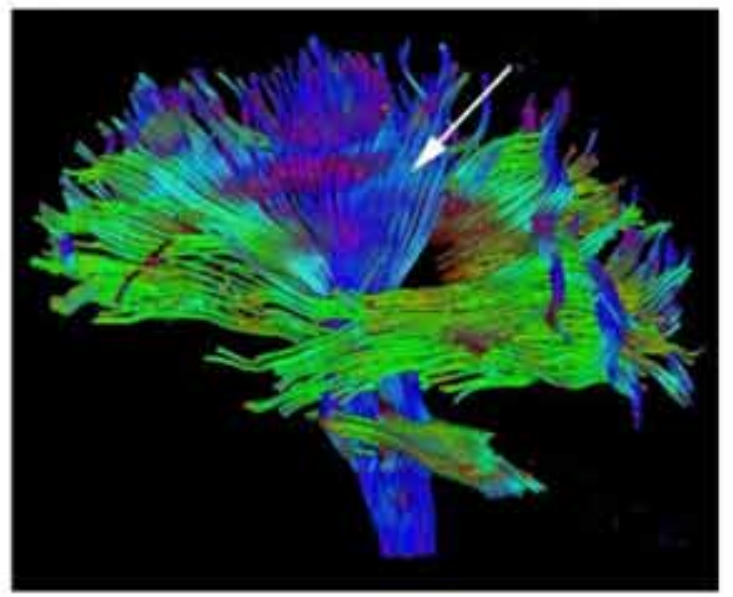

B

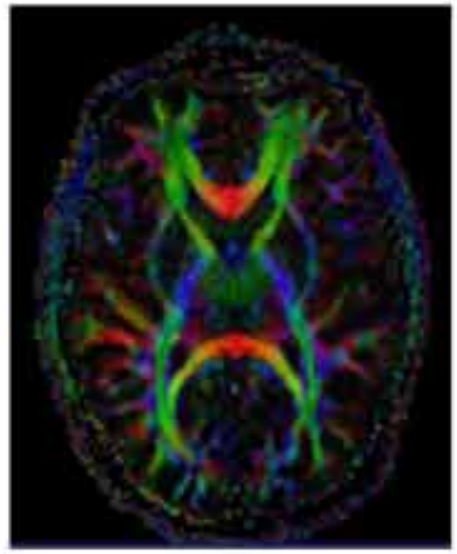

D

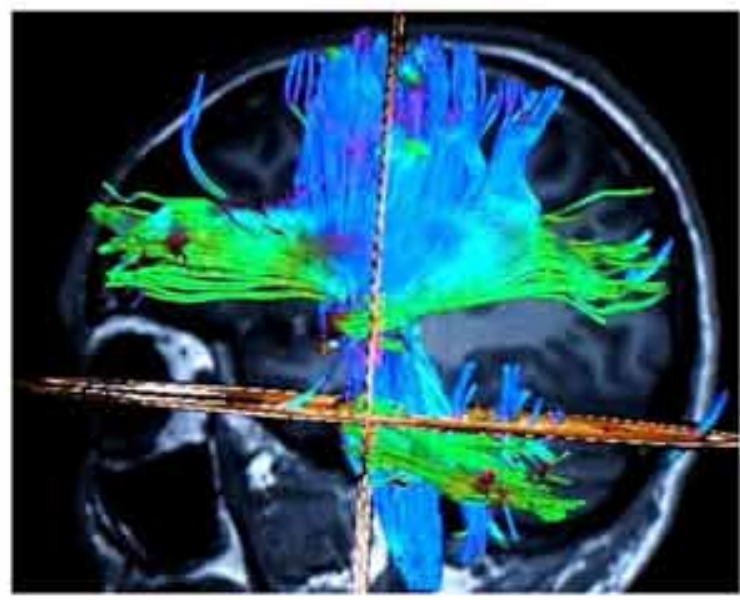

Figure 3: DTI scans of the athlete 1 year after last concussion. Three-dimensional DTI images were acquired in the axial plane parallel with anterior and posterior commisure axis covering the entire brain (Siemens MAGNETOM Spectra 3T Skyra). A single shot spin-echo echo planar imaging (EPI) was used for DTI acquisition with the following parameters: 44 slices without a gap, $F o V=230 \mathrm{~mm}$, slice thickness=2 mm, TR=5900 msec, TE=95 ms, bandwidth=1000 Hz, echo spacing=1 ms. For whole brain coverage, the total DTI scan time was about $15 \mathrm{~min}$. Fractional anisotropy (FA) map generated with syngo DTI Tractography for a healthy person (A) and a subject with concussion (B). White matter fiber tracts seen with syngo DTI Tractography (256 diffusion directions) in a healthy person (C) and a subject with concussion (D). 
The detection of baseline NR2 peptide in observed subjects showed that $12(14 \%)$ out 84 subjects had increased biomarker levels at baseline assessment (Figure $2 \mathrm{C}, 1$ ) that might be an indicator of transient ischemic edema in athletes after multiple concussions [71]. Two out of three concussed subjects with slightly increased NR2 peptide values $(0.9-1.0 \mathrm{ng} / \mathrm{mL}$ vs. cut off of $0.5 \mathrm{ng} / \mathrm{mL}$ ) showed reduced visual memory composite score by $5 \%$ and $21 \%$, respectively (defined in 2013 ImPACT testing). The monitoring of NR2 peptide levels in the same 3 subjects, (Figure 2D) whose AMPAR peptide data are discussed above, showed that only rugby athlete \# 3 had NR2 peptide levels steady increased during 3 months. This subject had a longer recovery time due to possible edema that was not seen on conventional 1.5T MRI [65] but might be determined by $3 \mathrm{~T}$ DWI/FLAIR analogue to recently reported cases [72].

\section{Concussions severity and persistent changes in brain}

Secondary injury mechanisms exacerbate tissue damage and BBB breakdown. The combination of primary and secondary injury releases antigens (structural proteins) that can drain into secondary lymphoid tissues, thereby activating neuroantigen-reactive $\mathrm{T}$ and $\mathrm{B}$ lymphocytes and producing antibodies. There are more than 100 antibodies against known antigens, most of which belong to CNS proteins [73].

Nervous tissue damage, vascular injury, and BBB disturbance facilitate output of brain antigens released from injured neurons that can activate the immune system [74]. Although most antibodies and lymphocytes are held in check by complex mechanisms of central and peripheral tolerance, their normal immune potential can be unleashed when the BBB is compromised [75]. It is plausible that if produced after brain injury, antibodies may be considered "natural," and bind CNS antigens without causing harmful consequences, including infiltration to the injured tissue [76,77]. Due to the nature of normal immune response, antibodies to natural antigens detected in serum are likely to be associated with advanced or secondary processes of brain damage [70].

Early investigations have established the capability of antibodies to GluR2/3 to enhance spiking activity in epilepsy $[78,79]$. Antibodies to the GluR1 subunit of AMPA receptors were also observed in patients with posttraumatic epilepsy $[80,81]$. Studies in children with chronic posttraumatic headaches as a result of multiple concussions have shown nonspecific brain spiking activity in $18 \%$ and epileptiform activity on EEG in 6\% [82]. Simultaneous detection of neurotoxicity biomarkers in serum samples of these patients demonstrated a $150 \%$ increase in N-terminal AMPA receptor antibodies.

The detection of baseline antibodies to N-terminal fragment of AMPAR peptide (AMPAR Ab) in observed subjects showed that 6 (7\%) out of 84 subjects had increased biomarker levels (Figure 4A) that might indicate the risk of brain spiking activity in these athletes. In longitudinal study, AMPAR Ab values remained abnormally high in the blood only athlete \#2 (Figure $4 \mathrm{~A}$ ) that may reflect persistent changes in the brain. The monitoring of AMPAR Ab levels in the same subject from baseline up to 15 months showed constant drastic elevation of the biomarker values (Figure 4B) accompanied with headaches and visual problems. This athlete had visual memory composite score decreased from baseline of $96 \%(08 / 16 / 2011)$ to $48 \%$ in ImPACT test performed on February 25, 2013. Athlete \#2 should be directed to neurologist for electroencephalography (EEG) performed and would need a treatment assignment.

Concussion also increases the risk of ischemic or hemorrhagic changes due to vascular system impairment, which may trigger future risk of strokes [83]. During a 5-year follow up clinical study, $8.2 \%$ of patients with TBI experienced stroke. It has been found that a "concussion penumbra" similar to the ischemic penumbra that occurs following an ischemic stroke [84].

Compared to baseline levels of NR2 peptide (acute phase marker), abnormally elevated concentrations of NR2 antibodies were observed in $29(34.5 \%)$ out of 84 subjects that is 2.5 times higher than that of the peptide values (Figure 4C). The latter might indicate persistent changes in the brain with delayed cerebral ischemia resembling to transient ischemic attack (TIA) that often is asymptomatic or symptoms are passing within an hour. In three subjects with prior concussion, NR2 antibody maintained increased levels beyond the cutoff of $2.0 \mathrm{ng} / \mathrm{mL}$ (Figure 4D). The monitoring of NR2 antibody levels in two out of 3 subjects showed that both had NR2 antibodies drastically elevated during entire study and accompanied with visual memory composite score reduced by $48 \%$ for both athletes in $2013 \mathrm{ImPACT}$ testing. These subjects should be assigned for combination of advanced DWI/FLAIR/ DTI by clinician to evaluate the presence of ischemic lesions [72].

Our pilot longitudinal study examined the diagnostic potential of neurotoxicity biomarkers in conjunction with neuropsychological testing and neuroimaging in small group of athletes to differentiate those with concussions in need of further neurological assessment and treatment. A body of advanced MRI-based analyses of transient or persistent changes in brain structures should be performed simultaneously with biomarker detection to estimate the preliminary correlation between damaged area and each biomarker assessed. A study of a subpopulation of subjects who presented early after concussion (within 3 hours) and who had rapid advanced imaging to gain a first glimpse into the relation between dynamic changes in brain tissue and its effect on biomarker levels would be needed.

\section{Three-circle concept in identification of concussion severity}

There is limited objective evidence [72] that mild impact leads to structural damage; however, it can trigger metabolic and functional disturbances, causing development of long-term secondary impairment in nervous tissue. There is a critical need to develop a strategy or "three-circle concept" [64] in assessment of suspected concussions and their severity that should include the following steps: (i) the primary neurocognitive assessment, (ii) blood assay detecting multiple biomarkers for early recognition of the brain metabolic and functional disturbances, and (iii) advanced neuroimaging modalities evaluating transient or persistent structural changes in brain areas. Based on this information a physician could choose the individual treatment approach that might attenuate or reverse brain injury consequences.

With the stated objective of rapid assessment of suspected concussions and post-concussion syndrome in sport and military settings, computerized tests have several limitations: (i) the performance of an unsupervised subject sitting in front of a PC may not always represent an optimal testing environment; (ii) tests can generate a mass of seemingly precise data, which clinical significance may be hard to interpret properly even for an experienced neuropsychologist; (iii) often players are missing baseline neurocognitive evaluation and continue to play even when subtle injury to the brain is present that likely will increase the risk of secondary damage accompanied with neurological symptoms. Then there are up to $80 \%$ of the general population admitted to ED with suspected acute concussions/mild TBI who usually reject or are unable to participate in cognitive testing due to feeling poorly and/or alcohol/drug influence. 
Citation: Dambinova SA, Sowell RL, Maroon JC (2013) Gradual Return to Play: Potential Role of Neurotoxicity Biomarkers in Assessment of Concussions Severity. J Mol Biomark Diagn S3: 003. doi:10.4172/2155-9929.S3-003

A

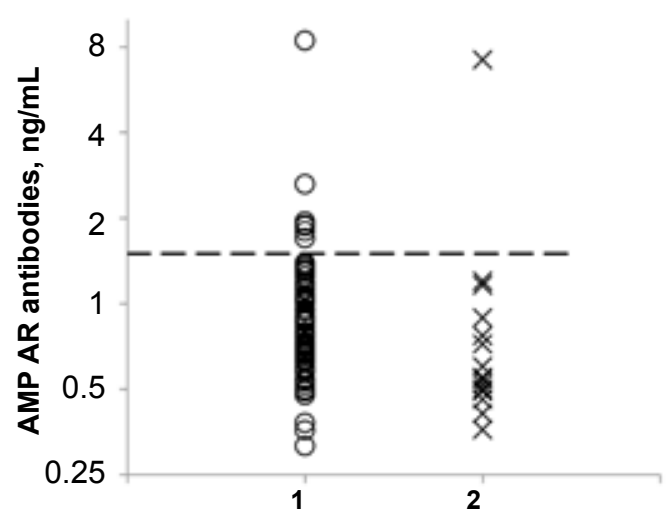

C

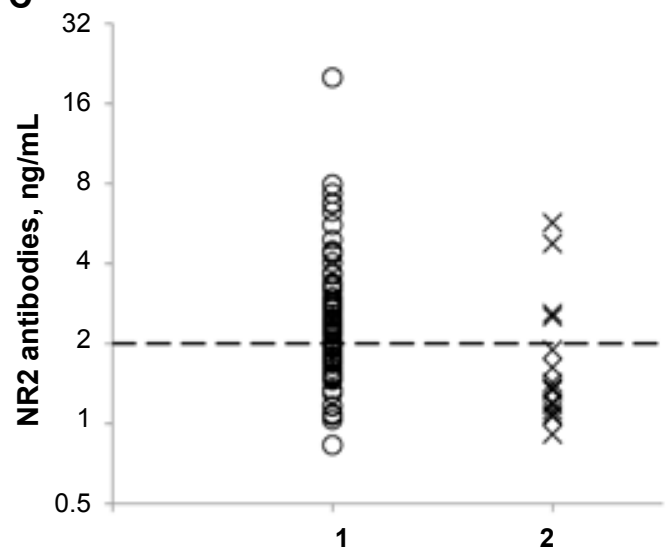

B

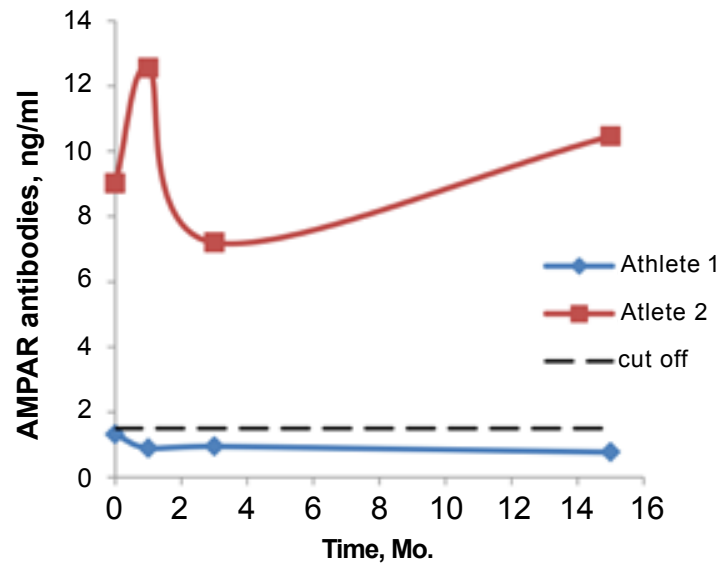

D

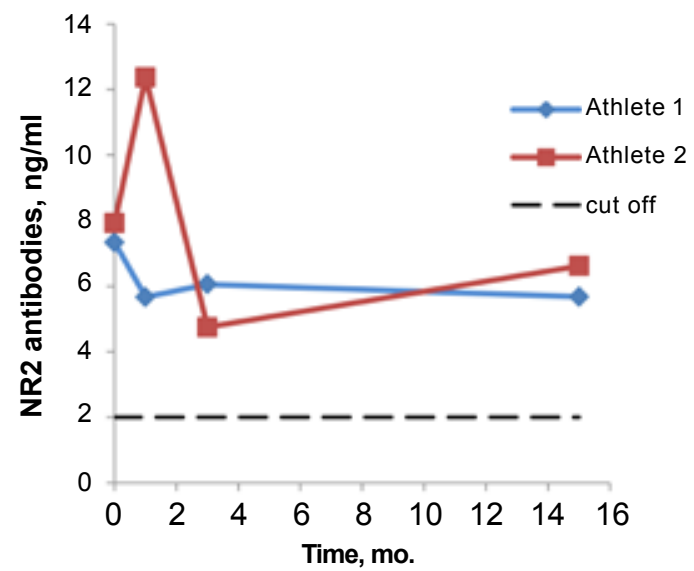

Figure 4: Distribution of serum AMPAR and NR2 antibodies respectively in A, C: (1) all enrolled athletes ( $n=84$ ); (2) subjects returned for longitudal study ( $\mathrm{n}=18$ ). Dynamic changes of AMPAR and NR2 antibodies levels in serumof subjects (B, D correspondently). Dotted lines show corresponding cut offs: $1.5 \mathrm{ng} / \mathrm{mL}$ for AMPAR antibodies and $2.0 \mathrm{ng} / \mathrm{mL}$ for NR2 antibodies.

Conventional CT and MRI that have a limited sensitivity to detect diffuse or traumatic axonal injury leaving concussion as a controversial diagnosis due to "normal" neuroimaging. Despite a significantly improved resolution of advanced MRI modalities, their use in assessment of mild TBI meets several challenges: (i) how objectively select persons with concussion or mild TBI for the MRI study out of $15-30 \%$ of those diagnosed on the basis of cognitive and clinical symptoms, (ii) what criteria should be used in asymptomatic cases, (iii) how increase turnaround time for MRI data acquisition and analyses, and what MRI modalities should be used in search of the area of subtle injury?

The primary transient effect of impact (often asymptomatic or subclinical) and delayed secondary structural damage (symptomatic) after the impact may be assessed by various biomarkers timely released into biological fluids. Perfect biomarker(s) for concussions in acute condition should (i) reflect the origin of transient subtle brain injury resulting from the impact, (ii) show a specific location of subtle brain injury, (iii) enter biological fluids at an early stage of brain damage (within minutes to hours after event), (iv) correlate with neurocognitive scores, and (v) navigate advanced neuroimaging (fMRI, DTI and SWI modalities) for possible areas of functional deficit or metabolic disturbances.

Biomarkers for chronic and delayed phase following impact(s) should (i) reflect neurological symptoms of persistent changes in CNS as a secondary injury, (ii) represent the immune system response to inflammation, (iii) predict outcome, (iv) navigate treatment, and adjustment of therapy. The secondary injury is usually symptomatic and results from pathophysiological consequences of primary impact after the initial trauma. It is often linked to edema, microvascular dysfunctions, compromised blood-brain barrier, delayed cerebral ischemia, and immunoexcitotoxicity. The search of real time perfect brain-born biomarker(s) for assessment of suspected concussions is challenging and holds a high priority in diagnosis of mild TBI.

To date GFAP and ICH-L1 could be considered as potential molecular biomarkers for assessment of acute mild to moderate TBI while Tau protein could be a marker of chronic traumatic encephalopathy resulting from multiple concussions. Despite high sensitivity or specificity found for the majority of biomarkers, limitations 
to study design and reporting prevented the recommendation of a specific biomarker for use in the clinical setting. Important questions that may be answered by future studies of brain biomarkers are those that address their feasibility in the management of concussions and mild TBI: whether a person sustained a concussion; whether a person will have an adverse outcome after concussion, and when an athlete can safely return to play. The role of current and emerging biomarkers should be considered from the practical stand point. They should substantiate and be reliable in making decisions on the field, at ED, primary physician office, or inpatient and outpatient settings.

Our studies suggest that biomarkers of neurotoxicity may assist in the assessment of transient changes in the brain due to acute and semiacute subtle or asymptomatic axonal injuries as well as structural changes associated with abnormal spiking activity or delayed ischemic edema. However, it is necessary to obtain a body of data using the "three-circle concept" to prove reliability of neurotoxicity biomarkers in evaluation of concussions. It is also a current need to develop an objective scoring system assessing the severity of concussions. These results could supply clinicians with the information concerning the brain status and will aid healthcare providers in making decisions about the choice of treatment. Additionally, data presented here demonstrated that the value of specific brain biomarkers detected in human biological fluids used in conjunction with advanced neuroimaging and neuropsychological scoring might assist in determining when an injured athlete should return to play.

\section{Conclusion}

One promising line of brain injury research would be use of both antigens and antibodies as biomarkers of neurotoxicity for assessment of TBI [63-66] stroke [67,70,71] and epilepsy [78,80,81]. Rapid assessment of subclinical CNS injuries using appropriate blood test(s) would be useful in various clinical settings, e.g., emergency departments and primary care offices as well as inpatient and outpatient facilities. Such tests could monitor treatment modalities and outcome. A rapid management of acute brain injuries is essential to ensure that patients receive proper treatment especially if the CT is normal or MRI is contraindicated or not available. The ability to hasten ruling in or ruling out axonal/neuronal or vascular damages prior to neuroimaging using a blood test(s) to detect a specific brain biomarker-or panel of biomarkers-would be extremely useful.

Timely assessment of concussions is essential in return to play making decision. Clinical use of blood tests that can reliably predict consequences, or forecast recovery and outcome, might help in selection of athletes for preventive therapy that could improve acute/ sub-acute health outcomes [85].

The latter would have the potential to shorten the often lengthy recovery process after mild head injuries and prevent further development of subsequent neurological complications. This would not only benefit the person with suspected concussion, but also assist the healthcare system by reducing direct and indirect costs.

\section{Acknowledgements}

The authors wish to acknowledge Laura St. Onge and Erika Walker, (Kennesaw State University Club Sports, Kennesaw, GA) for the help with sport club athletes enrollment, also thank Dr. Joseph D. Weissman, Dr. David Olson, Dr. John Reid (DeKalb Medical, Decatur, GA) for collaboration in MRI/DTI studies and Dr. John D. Mullins (Piedmont Hospital, Atlanta, GA) for consultations.

\section{Funding}

The study was supported by Well Star College of Health \& Human Services and in part by the Kennesaw State University Foundation. The funder's had no role in study design, data collection and analysis, decision to publish, or preparation of the manuscript.

\section{References}

1. Faul M, Xu L, Wald MM, Coronado VG (2010) Traumatic Brain Injury in the United States: Emergency Department Visits, Hospitalizations, and Deaths. Atlanta, GA, USA: Centers for Disease Control and Prevention, National Center for Injury Prevention and Control.

2. Biomarkers Definition Working Group: Biomarkers and Surrogate Endpoints: Prefferred Definitions and Conceptual Framework (2001) Clin Pharmacol The 69: 89-95.

3. Jeter CB, Hergenroeder GW, Hylin MJ, Redell JB, Moore AN, et al. (2012) Biomarkers for the diagnosis and prognosis of mild traumatic brain injury/ concussion. J Neurotrauma Oct 12.

4. Finnoff JT, Jelsing EJ, Smith J (2011) Biomarkers, genetics, and risk factors for concussion. PM R 3: S452-S459.

5. Dambinova SA (2012) Neurodegradomics: the source of biomarkers for mild traumati brain injury: Biomarkers for traumatic brain injury. RSC Publishing Cambridge.

6. Coppel DB (2011) Use of neuropsychological evaluations. Phys Med Rehabil Clin N Am 22: 653-664.

7. Van Kampen DA, Lovell MR, Pardini JE, Collins MW, Fu FH (2006) The "value added" of neurocognitive testing after sports-related concussion. Am J Sports Med 34: 1630-1635.

8. Randolph C (2011) Baseline neuropsychological testing in managing sportrelated concussion: does it modify risk? Curr Sports Med Rep 10: 21-26.

9. Lovell MR, Iverson GL, Collins MW, Podell K, Johnston KM, et al. (2006) Measurement of symptoms following sports-related concussion: reliability and normative data for the post-concussion scale. Appl Neuropsychol 13: 166-174.

10. Iverson GL, Lovell MR, Collins MW (2005) Validity of ImPACT for measuring processing speed following sports-related concussion. J Clin Exp Neuropsycho 27: 683-689.

11. SchatzP, PardiniJE, LovellMR, Collins MW, PodellK (2006) Sensitivity and specificity of the ImPACT Test Battery for concussion in. Arch Clin Neuropsychol 21: 91-99.

12. Barker JM, Wright DW, Goldstein FC, Ockerman J, Ratcliff JJ, et al. (2007) The DETECT system: portable, reduced-length neuropsychological testing for mild traumatic brain injury via a novel immersive environment. J Med EngTechno 31: 161-169.

13. Heitger MH, Jones RD, Macleod AD, Snell DL, Frampton CM, et al. (2009) Impaired eye movements in post-concussion syndrome indicate suboptimal brain function beyond the influence of depression, malingering or intellectual ability. Brain 132: 2850-2870.

14. Maruta J, Suh M, Niogi SN, Mukherjee P, Ghajar J (2010) Visual tracking synchronization as a metric for concussion screening. J Head Trauma Rehabil 25: 293-305

15. Wedeen VJ, Rosene DL, Wang R, Dai G, Mortazavi F, et al. (2012) The geometric structure of the brain fiber pathways.Science 335: 1628-1634.

16. Shenton ME, Hamoda HM, Schneiderman JS, Bouix S, Pasternak O, et al. (2012) A review of magnetic resonance imaging and diffusion tensor imaging findings in mild traumatic brain injury. Brain Imaging Behav 6: 137-192.

17. BelangerHG, Vanderploeg RD, Curtiss G, Warden DL (2007) Recen neuroimaging techniques in mild traumatic brain injury. J Neuropsychiatry Clin Neurosci 19: 5-20

18. Mayer AR, Ling J, Mannell MV, Gasparovic C, Phillips JP, et al. (2010) A prospective diffusion tensor imaging study in mild traumatic brain injury. Neurology 74: 643-650.

19. Mayer AR, Mannell MV, Ling J, Elgie R, Gasparovic C, et al. (2009) Auditory orienting and inhibition of return in mild traumatic brain injury: a FMRI study. Hum Brain Mapp 30: 4152-4166.

20. Talavage TM, Nauman E, Breedlove EL, Yoruk U, Dye AE, et al. (2013) Functionally-detected cognitive impairment in high school football players without clinically-diagnosed concussion. J Neurotrauma April 11.

21. Bigler ED, Bazarian JJ (2010) Diffusion tensor imaging: a biomarker for mild traumatic brain injury? Neurology 74: 626-627. 
Citation: Dambinova SA, Sowell RL, Maroon JC (2013) Gradual Return to Play: Potential Role of Neurotoxicity Biomarkers in Assessment of Concussions Severity. J Mol Biomark Diagn S3: 003. doi:10.4172/2155-9929.S3-003

Page 10 of 11

22. Mayer AR, Mannell MV, Ling J, Gasparovic C, Yeo RA (2011) Functional connectivity in mild traumatic brain injury. Hum Brain Mapp 32: 1825-1835.

23. Moffett JR, Tieman SB, Weinberger DR, Coyle JT, Namboodiri MA (2006) N-Acetylaspartate: A Unique Neuronal Molecule in the Central Nervous System. Springer Science + Business Media, New York.

24. Cohen BA, Inglese M, Rusinek H, Babb JS, Grossman RI, et al. (2007) Proton MR spectroscopy and MRI-volumetry in mild traumatic brain injury.AJNR Am J Neuroradiol 28: 907-913.

25. Johnson B, Gay M, Zhang K, Neuberger T, Horovitz SG, et al. (2012)The Use of magnetic resonance spectroscopy in the subacute evaluation of athletes recovering from single and multiple mild traumatic brain injury.J.Neurotrauma 29: $2297-2304$

26. Henry LC, Tremblay S, Leclerc S, Khiat A, Boulanger Y, et al. (2011) Metabolic changes in concussed American football players during the acute and chronic post-injury phases. BMC Neurol 23: 105-114.

27. Kirov II, Tal A, Babb JS, Reaume J, Bushnik T, et al. (2013) Proton MR spectroscopy correlates diffuse axonal abnormalities with post-concussive symptoms in mild traumatic brain injury.J Neurotrauma Jan 22.

28. Kou Z, Benson RR, Haacke EM (2011) Magnetic resonance imaging biomarkers of mild traumatic brain injury: Biomarkers for traumatic brain injury. RSC Publishing, Cambridge.

29. Iverson GL, Lange RT, Waljas M, Liimatainen S, Dastidar P et al. (2012) Outcome from Complicated versus Uncomplicated Mild Traumatic Brain Injury. Rehabil Res Pract 2012: 415740-415747.

30. Kou Z, Benson R, Gattu R, Yang J, Mika V (2011) Neuroimaging of mild TBI a acute stage. ProclntSoc Mag Res Med 19: 2449.

31. Blaylock RL, Maroon J (2011) Immunoexcitotoxicity as a central mechanism in chronic traumatic encephalopathy-A unifying hypothesis. Surg Neurol Int 2 : 107-132.

32. Marchi N, Bazarian JJ, Puvenna V, Janigro M, Ghosh C, et al. (2013) Consequences of repeated blood-brain barrier disruption in football players. PLoS One 8: e56805.

33. Hulsebosch CE (2008) Gliopathy ensures persistent inflammation and chronic pain after spinal cord injury. Exp Neurol 214: 6-9.

34. Dambinova SA (2012) Neurodegradomics: the source of biomarkers for mild TBI: Biomarkers for traumatic brain injury. RSC Publishing, Cambridge.

35. Neselius S, Brisby H, Theodorsson A, Blennow K, Zetterberg H, et al. (2012) CSF-biomarkers in Olympic boxing: diagnosis and effects of repetitive head trauma. PLoS One 7: e33606.

36. Papa L, Lewis LM, Falk JL, Zhang Z, Silvestri S, et al. (2012) Elevated levels of serum glial fibrillary acidic protein breakdown products in mild and moderate traumatic brain injury are associated with intracranial lesions and neurosurgical intervention. Ann Emerg Med 59: 471-483.

37. Metting Z, Wilczak N, Rodiger LA, Schaaf JM, van der Naalt J (2012) GFAP and $\mathrm{S} 100 \mathrm{~B}$ in the acute phase of mildtraumatic brain injury. Neurology 78 : 1428-1433.

38. Berger RP, Hayes RL, Richichi R, Beers SR, Wang KK (2012) Serum concentrations of ubiquitin C-terminal hydrolase-L1 and all-spectrin breakdown product $145 \mathrm{kDa}$ correlate with outcome after pediatric TBI. J Neurotrauma 29: 162-167.

39. Bazarian JJ, Zemlan FP, Mookerjee S, Stigbrand T (2006) Serum S-100B and cleaved-tau are poor predictors of long-term outcome after mild traumatic brain injury. Brain Inj 20: 759-765.

40. Ma M, Lindsell CJ, Rosenberry CM, Shaw GJ, Zemlan FP (2008) Serum cleaved tau does not predict postconcussion syndrome after mild traumatic brain injury. Am J Emerg Med 26: 763-768.

41. Neselius S, Zetterberg H, Blennow K, Randall J, Wilson D, et al. (2013) Olympic boxing is associated with elevated levels of the neuronal protein tau in plasma. Brain Inj 27: 425-433.

42. McKee AC, Stein TD, Nowinski CJ, Stern RA, Daneshvar DH, et al. (2013) The spectrum of disease in chronic traumatic encephalopathy. Brain 136: 43-64.

43. Papa L, Lewis LM, Silvestri S, Falk JL, Giordano P, et al. (2012) Serum levels of ubiquitin $\mathrm{C}$-terminal hydrolase distinguish mild traumatic brain injury from trauma controls and are elevated in mild and moderate traumatic brain injury patients with intracranial lesions and neurosurgical intervention. J Trauma Acute Care Surg 72: 1335-1344.

44. Pelkey K, McBain CJ (2008) lonotropic glutamate receptors in synaptic plasticity: The Glutamate Receptors. Humana Press, Totowa.

45. Sharp CD, Fowler M, Jackson TH, Houghton J, Warren A, et al. (2003) Human neuroepithelial cells express NMDA receptors. BMC Neurosci 4: 28-32.

46. Bergles DE, Jabs R, Steinhauser C (2010) Neuron-glia synapses in the brain Brain Res Rev 63: 130-137.

47. Gill S, Pulido O (2010) Glutamate receptors in peripheral tissues.Distribution and implications for toxicology:Glutamate Receptors in Peripheral Tissue. Excitatory Transmission Outside the CNS. Kluwer Academic Publishers, New York.

48. Ashby MC, Daw MI, Issac JT (2008) AMPA receptors: The Glutamate Receptors. Humana Press, Totowa.

49. Petralia RS, Wenthold RJ (2008) NMDA receptors: The Glutamate Receptors Humana Press, Totowa.

50. Jin XT, Smith $Y(2011)$ Localization and functions of kainite receptors in the basal ganglia: Kainate Receptors. Adv Exp Med Biol 717: 27-37.

51. Lai TW, Shyu WC, Wang YT (2011) Stroke intervention pathways: NMDA receptors and beyond. Trends Mol Med17: 266-275.

52. Prudova A, auf dem Keller U, Butler GS, Overall CM (2010) Multiplex $\mathrm{N}$-terminome analysis of MMP-2 and MMP-9 substrate degradomes by iTRAQTAILS quantitative proteomics. Mol Cell Proteomics 9: 894-911.

53. Stirling DP, Stys PK (2010) Mechanisms of axonal injury: internodal nanocomplexes and calcium deregulation. Trends Mol Med 16: 160-170.

54. Tekkök SB, Goldberg MP (2001) AMPA/kainate receptor activation mediates hypoxic oligodendrocyte death and axonal injury in cerebral white matter. $J$ Neurosci 21: 4237-4248.

55. Hammond JC, McCullumsmith RE, Funk AJ, Haroutunian V, Meador-Woodruff $\mathrm{JH}(2010)$ Evidence for abnormal forward trafficking of AMPAreceptors in fronta cortex of elderly patients with schizophrenia.Neuropsychopharmacology 35 2110-2119.

56. Lai M, Hughes EG, Peng X, Zhou L, Gleichman AJ, et al. (2009) AMPA recepto antibodies in limbic encephalitis alter synaptic receptor location. Ann Neurol 65 424-434.

57. Beneyto M, Meador-Woodruff JH (2004) Expression of transcripts encoding AMPA receptor subunits and associated postsynaptic proteins in the macaque brain. J Comp Neurol 468: 530-554.

58. Shrey DW, Griesbach GS, Giza CC (2011) The pathophysiology of concussions in youth. Phys Med RehabilClin N Am 22: 577-602.

59. Cull-Candy S, Brickley S, Farrant M (2001) NMDA receptor subunits: diversity, development and disease. Curr Opin Neurobiol 11: 327-335.

60. Betzen C, White R, Zehendner CM, Pietrowski E, Bender B, et al. (2009) Oxidative stress upregulates the NMDA receptor on cerebrovascula endothelium. Free Radical Biol Med 47: 1212-1220.

61. Singh P, Doshi S, Spaethling JM, Hockenberry AJ, Patel TP et al. (2012 $\mathrm{N}$-methyl-D-aspartate receptor mechanosensitivity is governed by $\mathrm{C}$ terminus of NR2B subunit. J Biol Chem 287: 4348-4359.

62. Spaethling J, Le L, Meaney DF (2012) NMDA receptor mediated phosphorylation of GluR1 subunits contributes to the appearance of calcium-permeable AMPA receptors after mechanical stretch injury. Neurobiol Dis 46: 646-654.

63. Danilenko UI, Khunteev GA, Bagumyan A, Izykenova GA (2012) Neurotoxicity biomarkers in experimental acute and chronic brain injury: Biomarkers for traumatic brain injury. RSC Publishing, Cambridge.

64. Dambinova SA, Gill S, St. Onge L, Sowell R (2012) Biomarkers for subtle brain dysfunction: Biomarkers for traumatic brain injury. RSC Publishing, Cambridge.

65. Dambinova SA, Shikuev AV, Weissman JD, Mullins JD (2013) AMPAR peptide values in blood of nonathletes and club sport athletes with concussions. Military Med 178: 285-290.

66. Dambinova SA (2007) Diagnostic challenges in traumatic brain injury. IVD Technology 3: 3-7

67. Dambinova SA (2008) Biomarkers for transient ischemic attack (TIA) and ischemic stroke. Clin Lab Inter 32: 7-11. 
Citation: Dambinova SA, Sowell RL, Maroon JC (2013) Gradual Return to Play: Potential Role of Neurotoxicity Biomarkers in Assessment of Concussions Severity. J Mol Biomark Diagn S3: 003. doi:10.4172/2155-9929.S3-003

68. Nag S, Kapadia A, Stewart DJ (2011) Review: molecular pathogenesis of bloodbrain barrier breakdown in acute brain injury. Neuropathol Appl Neurobiol 37: 3-23.

69. Doeuvre L, Plawinski L, Toti F, Angle's-Cano E (2009) Cell-derived microparticles: a new challenge in neuroscience. J Neurochem 110: 457-468.

70. Weissman JD, Khunteev GA, Heath R, Dambinova SA (2011)NR2 antibodies: risk assessment of transient ischemic attack (TIA)/stroke in patients with history of isolated and multiple cerebrovascular events. J Neurol Sci300: 97-102.

71. Dambinova SA, Bettermann K, Glynn T, Tews M, Olson D, et al. (2012) Diagnostic potential of the NMDA receptor peptide assay for acute ischemic stroke. PLoS One 7: e42362.

72. Parikh G, Ray-Chaudhury A, Latour L (2013) Brain imaging after mild head injury/concussion can show lesions. Proceedings of AAN $65^{\text {th }}$ meeting.

73. Pleasure D (2008) Diagnostic and pathogenic significance of glutamate receptor autoantibodies. Arch Neurol 65: 589-592.

74. Zhang Y, Popovich P (2011) Roles of autoantibodies in central nervous system injury.Discov Med11: 395-402.

75. Basten A, Silveira PA (2010) B-cell tolerance: mechanisms and implications. Curr Opin Immunol 22: 566-574.

76. Ehrenstein MR, Notley CA (2010)The importance of natural IgM: scavenger, protector and regulator. Nat Rev Immunol10: 778-786.

77. Wright BR, Warrington AE, Edberg DD, Rodriguez M (2009) Cellular mechanisms of central nervous system repair by natural autoreactive monoclonal antibodies. Arch Neurol 66: 1456-1459.
78. Rogers SW, Andrews PI, Gahring LC, Whisenand T, Cauley K, et al. (1994) Autoantibodies to glutamate receptor GluR3 in Rasmussen's encephalitis. Science 265: 648-651.

79. Twyman RE, Gahring LC, Spiess J, Rogers SW (1995) Glutamate recepto antibodies activate a subset of receptors and reveal an agonist binding site. Neuron 14: 755-762.

80. Odinak MM, Dyskin DE, Toropov IA, Emelin AY, Cherepanov AA, et al. (1996) The diagnosis of epilepsy by using blood analysis for the content of autoantibodies to glutamate neuroreceptor. Zh Nevrol Psikhiatr Im S S Korsakova 96: 45-48.

81. Gromov SA, Khorshev SK, PoliakovYul, Gromova LG, Dambinova SA (1997) Clinico-biochemical study in epilepsy. Zh Nevrol Psikhiatr Im S S Korsakova 97: 46-49.

82. Goryunova AV, Bazarnaya NA, Sorokina EG, SemenovaNYu, Globa OV, et al. (2007) Glutamate receptor autoantibody concentrations in children with chronic post-traumatic headache. Neurosci Behav Physiol 37: 761-764.

83. Chen $\mathrm{YH}$, Kang JH, Lin HC (2011) Patients with traumatic brain injury: population-based study suggests increased risk of stroke. Stroke 42: 2733 2739.

84. McKeag DB, Kutcher JS, Concensus C (2009) Raising the bar and filling in the gaps. Clin J Sports Med 19: 343-346.

85. Bailey N (2011) The perspectives of an R.D. working with civilian traumatic brain injury: Nutrition and Traumatic Brain Injury. The National Academies Press, Washington, DC. 